\title{
Putative Phospholipase B-like 2 (PLBD2) is not responsible for polysorbate degradation in monoclonal antibody drug products
}

\author{
sisi Zhang ${ }^{1}$, hui xiao ${ }^{1}$, michael Goren ${ }^{1}$, Darya Burakov ${ }^{1}$, Gang chen ${ }^{1}$, and Ning Li $^{1}$ \\ ${ }^{1}$ Regeneron Pharmaceuticals Inc
}

May 5, 2020

\begin{abstract}
Polysorbates (PS) are surfactants commonly added in a therapeutic protein drug product as excipients to protect proteins from denaturation and aggregation during storage, transportation, and delivery. Significant degradation of PS in drug products could lead to shortened drug shelf lives and PS-degrading activity in drug products must be minimized. Identification of lipases that degrade PS could lead to better process control in drug manufacturing. In 2016, phospholipase B-like 2 (PLBD2) was proposed as a residual host cell protein responsible for degrading PS20 in a drug formulation. We have carried out a series of studies to verify the role of PLBD2 in degrading polysorbates in drug products purified from recombinant Chinese Hamster Ovary (CHO) cells. Genetic knock-out and immuno-depletion results showed that when PLBD2 was removed or depleted, the degradation of PS20 or PS80 was neither diminished nor reduced. In addition, a quantitative analysis of PLBD2 and PS20 degradation in multiple formulated mAb products did not establish a correlation between the amount of PLBD2 and the level of PS20 degradation. Collectively these results suggest that PLBD2 is not the primary cause of polysorbate degradation in formulated drug products purified using standard Protein A and ion exchange chromatography.
\end{abstract}

\section{Introduction}

PS20 and PS80 are the most commonly used surfactants in biopharmaceutical protein formulation that can improve protein stability and protect drug products from aggregation and denaturation during delivery and transportation (Kiese, Papppenberger, Friess, \& Mahler, 2008; Martos et al., 2017) . However, polysorbate is labile to hydrolysis and the hydrolyzed products can lead to the formation of undesired particulates in the formulated drug substances(Hall, Sandefur, Frye, Tuley, \& Huang, 2016). The mechanism of hydrolysis of polysorbates had been widely studied by multiple groups across industry. In addition to acid and baseinduced polysorbate hydrolysis, host cell derived lipases or esterase co-purified with the final drug substance have also been shown to degrade polysorbate, with distinct degradation profiles unique to PS20 or PS80. For example, porcine liver esterase was reported to be able to specifically hydrolyze PS80 but not PS20, resulting in the formation of PS85 over time in mAb drug product(Labrenz, 2014). Group XV lysosomal phospholipase A2 isomer X1 (LPLA2) demonstrated the ability to degrade monoesters in both PS20 and PS80 at a concentration less than 1 ppm(Cheng et al., 2019; Hall et al., 2016). Unique degradation pattern in both PS20 or PS80 was found in a range of carboxylesters, including pseudomonas cepacia lipase on immobead 150 (PCL), candida antarctica lipase B on immobead 150 (CALB), thermomyces lanuginosus lipase on immobead 150 (TLL), rabbit liver esterase (RLE), Candida antarctica lipase B (CALB) and porcine pancreatic lipase type II (PPL)(McShan, Kei, Ji, Kim, \& Wang, 2016).

In 2016, phospholipase B-like 2 (PLBD2) was first reported as the residual host cell protein present in a sulfatase drug product that can degrade polysorbate 20 over time(Dixit, Salamat-Miller, Salinas, Taylor, \& Basu, 2016). That conclusion was made based on the experiment were PS20 degradation can be accelerated upon spiking in the recombinant human PLBD2 protein. However, as the recombinant PLBD2 used in the study only had a purity of $\sim 90 \%$, the enzymatic contribution from impurities could not be ruled out. 
In this study, we engineered a PLBD2 knockout CHO host cell line. Several monoclonal antibodies were expressed from the PLBD2 knockout $\mathrm{CHO}$ and wild type CHO host cell lines. The purified mAbs from the PLBD2 knockout cell line showed no significant difference in lipase activity compared to the same product produced from the control CHO cell line with wild type PLBD2. Consistent with this result, the removal of PLBD2 protein in mAbs purified from wild type CHO by immuno depletion with an anti-PLBD2 antibody did not significantly decrease lipase activity. In addition, our PLBD2 quantitation analysis of multiple formulated $\mathrm{mAb}$ products showed no correlation between the amount of PLBD2 in the sample and the extent of PS20 degradation. Taken together, our data suggest that PLBD2 itself does not contribute significantly to polysorbate degradation.

Proteomics analysis of the commercially available recombinant PLBD2 identified several additional lipases, providing a likely explanation for the initial misidentification of PLBD2 as the root course of PS20 degradation.

\section{Materials and Methods}

Materials

WedgeWell Tris-Glycine 4-12\% Mini Gels, SeeBlue Plus2 pre-stained molecular weight standards and $1 \mathrm{M}$ Tris-HCl (pH 8) were purchased from Invitrogen of Thermo Fisher Scientific (Waltham, MA). Dynabeads MyOne Streptavidin T1 and Dynabeads Antibody Coupling Kit was purchased from Invitrogen of Thermo Fisher Scientific (Waltham, MA). Trans-Blot Turbo Transfer Pack was purchased from Bio-Rad (Hercules, CA). Formic acid, acetonitrile, Diothiothreitol (DTT) and 1-step ultra TMD-blotting solution were purchased from Thermo Fisher Scientific (Waltham, MA). Acetic acid, 10X Tris buffered saline (TBS), Iodoacetamide (IAM), bovine serum albumin (BSA) and urea were purchased from Sigma-Aldrich (St. Louis, MO). HEPES buffered saline with EDTA and 0.005\% v/v Surfactant P-20 (HBS-EP) was purchased from GE (Boston, MA). All monoclonal antibodies, recombinant CHO PLBD2, anti-PLBD2 monoclonal antibody were prepared in house at Regeneron Pharmaceutical. Inc. Polysorbate 20 and polysorbate 80 were purchased from Croda and aliquoted in Regeneron Pharmaceutical. Inc. Human PLBD2 was purchased from Origene Technologies Inc (Rockville, MD). Sequencing Grade Modified Trypsin was purchased from Promega (Madison, WI). Antigoat IgG antibody was purchased from Abcam (Cambridge, UK). Oasis Max column, Acquity UPLC BEH C4 column, Acquity UPLC CSH C18 column were purchase from Waters (Milford, MA). Acclaim PepMap 100 column and Acclaim PepMap RSLC column were purchased from Thermo Fisher Scientific (Waltham, MA). DPBS (10x) was purchased from Gibco by life technologies and Tween20 was purchased from J.T. Baker (Phillipsburg, NJ). Q-Exactive Plus with electrospray ionization (ESI) source was purchased from Thermo Fisher Scientific (Waltham, MA).

Antibody mAb-1 is an IgG4 antibody expressed from a CHO K1 cell line; mAb-2 is the same IgG4 antibody as mAb-1 but expressed from a CHO cell line harboring a PLBD2 gene knocked out; mAb-3 is mAb-1 processed, as described below, to remove PLBD2; mAb-4, mAb-5, mAb-6 and mAb-7 are different IgG4 antibodies without the PLBD2 removal purification step; mAb-8 is an IgG1 antibody without the PLBD2 removal step.

Two-Dimensional Liquid Chromatography- Charged Aerosol detector (CAD)/ Mass Spectrometry (MS) Assay to Analyze Polysorbate Degradation

Degradation of PS20 and PS80 in CHO cell-free media or formulated antibody were analyzed by twodimensional HPLC-CAD/ MS system. The details of the setup were previously described by Genentech (Li et al., 2014). Polysorbates were separated from formulated $\mathrm{mAb}$ by using an Oasis MAX column $(2.1 \times 20$ $\mathrm{mm}, 30 \mu \mathrm{m})$, with the initial gradient held at $99 \%$ solvent A $(0.1 \%$ formic acid in water $)$ and $1 \%$ solvent B ( $0.1 \%$ formic acid in acetonitrile) for $1 \mathrm{~min}$. The gradient was then increased to $20 \%$ solvent B in 1.5 min and dropped back down to $1 \%$ in another $1.5 \mathrm{~min}$. The up and down gradient cycle for solvent B was repeated 3 times over 10 min to achieve the complete removal of $\mathrm{mAb}$ from the polysorbates. By using a switch valve, polysorbates were then subjected to separation by reversed phase chromatography using Acquity BEH C4 column $(2.1 \times 50 \mathrm{~mm}, 1.7 \mu \mathrm{m})$. During the separation, solvent B was increased to $20 \%$ from $1 \%$ in $1.5 \mathrm{~min}$ at 
the end of the 10 min gradient cycle, then gradually increased to $99 \%$ at $45 \mathrm{~min}$ and held for $5 \mathrm{~min}$, followed by an equilibration step of $1 \% \mathrm{~B}$ for $5 \mathrm{~min}$. The flow rate was kept at $0.1 \mathrm{~mL} / \mathrm{min}$ and column temperature at $40^{\circ} \mathrm{C}$.

The 2D-LC system was set up with Thermo UltiMate 3000 and coupled with Corona Ultra CAD detector operated under a nitrogen pressure of 75 psi for quantitation. Chromeleon 7 was used for system control and data analysis. Q-Exactive Plus with ESI source was coupled with the 2D-LC system for characterization only. The instrument was operated in a positive mode with capillary voltage at $3.8 \mathrm{kV}$, capillary temperature at $350^{\circ} \mathrm{C}$, sheath flow rate at 40 , and aux flow rate at 10 . Full scan spectra were collected over the $\mathrm{m} / \mathrm{z}$ range of 150-2000. Thermo Xcalibur software was used to collect and analyze MS data.

Peak area of each ester was integrated from the CAD chromatogram and summed up to account for the total intact ester in PS20 or PS80. Because peak areas of diesters do not change during incubation, only the sum of peak areas of monoesters were used for calculations. The remaining percentage of PS20 used in this work was calculated by comparing the sum of the peak areas of monoesters eluting between $27.5 \mathrm{~min}$ and $33 \mathrm{~min}$ at each time point to the sum of peak areas at time zero. The remaining percentage of PS80 was calculated by comparing the sum of the peak areas of monoesters eluting between $30 \mathrm{~min}$ and $35 \mathrm{~min}$ at each time point to the sum of peak areas at time zero.

Hydrolysis of Polysorbate 20 and PS80 with Putative Phospholipase B-like 2 (PLBD2) and Formulated Antibody

To evaluate the effect of PLBD2 on PS20 and PS80 degradation, $16 \mu \mathrm{L}$ of $200 \mathrm{mg} / \mathrm{mL} \mathrm{mAb}-3$ in $10 \mathrm{mM}$ Histidine buffer pH 6.0 was mixed with $2 \mu \mathrm{L}$ of $1 \%$ PS20 or $1 \%$ PS 80 , followed by adding $2 \mu \mathrm{L}$ of $0.2 \mathrm{mg} / \mathrm{mL}$ PLBD2 and incubating the sample at $45^{\circ} \mathrm{C}$ for 5 days. One aliquot $(3 \mu \mathrm{L})$ of each sample was diluted 25 -fold by adding $72 \mu \mathrm{L} 10 \mathrm{mM}$ histidine, $\mathrm{pH} 6.0$ and used for LC-CAD analysis.

The hydrolysis of PS20 in formulated mAb was examined by mixing $18 \mu \mathrm{L}$ of $75 \mathrm{mg} / \mathrm{mL} \mathrm{mAb}$ (buffer exchanged to $10 \mathrm{mM}$ Histidine, $\mathrm{pH}$ 6.0) with $2 \mu \mathrm{L}$ of $1 \%$ PS20 followed by incubation at $45^{\circ} \mathrm{C}$ for 5 days. The hydrolysis of PS80 in formulated mAb was examined by mixing $18 \mu \mathrm{L}$ of $100 \mathrm{mg} / \mathrm{mL} \mathrm{mAb}$ (buffer exchanged to $10 \mathrm{mM}$ Histidine, $\mathrm{pH} 6.0$ ) with $2 \mu \mathrm{L}$ of $1 \% \mathrm{PS} 80$ followed by incubation at $45^{\circ} \mathrm{C}$ for 5 days. One aliquot $(3 \mu \mathrm{L})$ of each sample was diluted 25 -fold by $10 \mathrm{mM}$ Histidine, $\mathrm{pH} 6$ and used for LC-CAD analysis.

\section{LC-multiple reaction monitoring (MRM) quantitation of PLBD2}

Both purified antibody mAb-3 with spiked-in PLBD2 standard and mAb drug substance (mAb-3, mAb-4, $\mathrm{mAb}-5, \mathrm{mAb}-6, \mathrm{mAb}-7, \mathrm{mAb}-8$ ) were digested by trypsin and then were subjected to LC-MRM analysis. LC-MRM analysis was performed on an Agilent 6495A QQQ Mass Spectrometry (Wilmington, DE) equipped with an Agilent 1290 infinity HPLC (Wilmington, DE). $20 \mu \mathrm{L}$ of the digested samples were injected onto an Acquity BEH C18 column $(2.1 \times 50 \mathrm{~mm}, 1.7 \mu \mathrm{m})$ at $40^{\circ} \mathrm{C}$ pre-equilibrated with $12 \%$ mobile phase B $(0.1 \%$ formic acid in acetonitrile, mobile phase $\mathrm{A}$ is $0.1 \%$ formic acid in water) at a flow rate of $0.4 \mathrm{~mL} / \mathrm{min}$. Post sample injection the gradient was maintained isocratically at $12 \% \mathrm{~B}$ for $0.5 \mathrm{~min}$, followed by a linear increase to $15 \% \mathrm{~B}$ over $6 \mathrm{~min}$, and then increased to $90 \% \mathrm{~B}$ in $0.1 \mathrm{~min}$ after which the gradient was kept at $90 \% \mathrm{~B}$ for $2.5 \mathrm{~min}$. In the end the gradient was decreased to $12 \% \mathrm{~B}$ to allow the column to be re-equilibrated for 3 min. Eluent between 2-13 min was analyzed using an ESI source operating under positive mode, with gas temperature $250^{\circ} \mathrm{C}$, gas flow $12 \mathrm{~L} / \mathrm{min}$, nebulizer gas $20 \mathrm{psi}$, sheath gas temperature $300^{\circ} \mathrm{C}$, sheath gas flow $11 \mathrm{~L} / \mathrm{min}$, capillary voltage $3500 \mathrm{~V}$ and nozzle voltage $500 \mathrm{~V}$. PLBD2 were monitored at $615.35 /$ 817.41 (SVLLDAASGQLR) for quantitation and 427.7/ 450.3 (YQLQFR) for confirmation. Peak integration was performed by Skyline(MacLean et al., 2010), and PLBD2 concentrations were calculated based on the calibration curved created by spiked-in PLBD2 standards.

\section{Western Blot of PLBD2}

Western blot was performed to confirm the existence of PLBD2. Samples were prepared by mixing $12.5 \mu \mathrm{L}$ mAb-1 $(4 \mathrm{mg} / \mathrm{mL})$ with $2.5 \mu \mathrm{L} 0.25$ M IAM and $10 \mu \mathrm{L} 2 \mathrm{x}$ Tris-Glycine loading buffer, followed by heating at $80^{\circ} \mathrm{C}$ for 2 min. $20 \mu \mathrm{L}$ sample was loaded onto the SDS-PAGE gel for electrophoresis separation at $160 \mathrm{~V}$ 
for $1.5 \mathrm{hrs}$, and the separated proteins were transferred to PVDF membrane at 25V for $30 \mathrm{~min}$. The PVDF membrane was then blotted by $2 \%$ BSA in PBST for $1 \mathrm{hr}$ at room temperature, followed by adding antiPLBD2 monoclonal antibody in $1 \%$ BSA $(1: 1000)$ and incubating at $4^{\circ} \mathrm{C}$ overnight. After washing with PBST three times, the secondary antibody anti-goat IgG was added at 1:5000 at room temperature for 1 hr. The PVDF membrane was then washed by PBST three times and stained by 1-step ultra TMD-blotting solution.

\section{Depletion of PLBD2 from CHO-Derived Antibodies}

PLBD2 depletion experiment was performed by using the Dynabeads antibody coupling kit as described by the manufacturer (Figure 5). Briefly, $5 \mathrm{mg}$ of pre-washed magnetic Dynabeads were mixed with $100 \mu \mathrm{g}$ anti-PLBD2 $\mathrm{mAb}$ in buffer $\mathrm{C} 1$. After addition of and buffer $\mathrm{C} 2$, the mixture was incubated overnight with gentle rocking at $4^{\circ} \mathrm{C}$. Beads were washed by $\mathrm{HB}, \mathrm{LB}$ and $\mathrm{SB}$ from the kit and then resuspend into $500 \mu \mathrm{L}$ water. $50 \mu \mathrm{L}$ resuspended anti-PLBD2 Dynabeads was added to each $10 \mathrm{mg} \mathrm{mAb}$ samples to a total volume of $500 \mu \mathrm{L}$ respectively, followed by shaking at room temperature for 3 hours. After removing the beads, the supernatant was dried under SpeedVac and resuspended into water. Protein concentration of mAb was measured and adjusted to $75 \mathrm{mg} / \mathrm{mL}$ for incubation with $0.1 \%$ PS20 and $100 \mathrm{mg} / \mathrm{mL}$ for incubation with $0.1 \%$ PS80.

Shotgun proteomics analysis of PLBD2

Both human and CHO PLBD2 samples were subjected to shotgun proteomics analysis. $10 \mu \mathrm{g}$ of PLBD2 was dried with Speedvac, then re-constituted with $20 \mu$ of denature/reduction buffer containing $8 \mathrm{M}$ urea and 10 $\mathrm{mM}$ DTT. The proteins were denatured and reduced at $37^{\circ} \mathrm{C}$ for $30 \mathrm{~min}$, and then incubated with $6 \mu \mathrm{l}$ of 50 $\mathrm{mg} / \mathrm{ml}$ iodoacetamide for $30 \mathrm{~min}$ in dark. Alkylated proteins were digested overnight with $50 \mu \mathrm{l} 0.01 \mu \mathrm{g} / \mu \mathrm{L}$ trypsin at $37{ }^{\circ} \mathrm{C}$. The peptide mixture was acidified by $5 \mu \mathrm{L}$ of $10 \%$ TFA. The sample was injected $10 \mu \mathrm{L}$ for LC-MS/MS analysis.

PLBD2 knockout cell line generation

In order to knock out PLBD2 gene in CHO K1 using CRISPR/Cas9, a small guide RNA (sgRNA) sequence corresponding to Exon 1 of PLBD2 was selected for specific targeting of PLBD2 exons 1. Sense (5'-tgtatgagaccacGCCCCCATGGACCGGAGCCC-3') and antisense (5'- aaacGGGCTCCGGTCCATGGG GCgtggtctca-3') oligonucleotides were ordered, with appropriate overhangs for cloning into CAS940A-1 (System Biosciences). The paired oligonucleotides were annealed at $5 \mu \mathrm{M}$ by incubation at $95{ }^{\circ} \mathrm{C}$ for 5 min followed by cooling to room temperature gradually. The annealed oligos were diluted 10x in water and ligated into CAS940A-1 using T4 DNA ligase (ThermoFisher Scientific, Waltham, MA). After transformation of Electromax DH10B cells (ThermoFisher Scientific, Waltham, MA), colonies were screened by sequencing. Maxi-preps of sequence verified plasmids containing PLBD2 sgRNA 1 was generated using the EndoFree Plasmid Maxi Kit (Qiagen).

CHO K1 cells were transfected with 5 ug of the CAS940A-1 plasmid encoding the PLBD2 sgRNA. The next day, the cells were harvested, and single cell sorted into 4 96-well plates containing Optipro-SFM by flow cytometry. After expansion, individual cell clones were analyzed by quantitative PCR (qPCR) for the disruptions in both genomic DNA (gDNA) and complementary DNA (cDNA). Double allelic disruption was confirmed by PCR and sequencing. Lack of PLBD2 expression was further confirmed by Western blot analysis.

\section{Results and Discussion}

Polysorbate in formulated $\mathrm{mAb}$ detected by 2D-LC-CAD/MS

Polysorbate in formulated mAbs were detected and identified by 2D-LC-CAD/ MS following a slightly modified method by Yi Li, et.al (Borisov, Ji, Wang, Vega, \& Ling, 2011; Li et al., 2014). As this study was concerned with hydrolysis of polyoxyethylene (POE) ester bonds, the gradient was set to retain all forms of POE esters while eliminating most POE, POE sorbitan, and POE isosorbide. Additionally, mAbs 
were eliminated using an Oasis Max Column. Reverse phase chromatography was then used to separate the remaining POE esters based on their fatty acid content and type. The esters eluted in the order of monoesters, diesters, triesters and tetraesters (Figure 1-II-V). The structure of each ester was elucidated by mass spectrometry based on the chemical formula of the polymer and dioxolanylium ion generated by in source fragmentation. Figure 1-III is the representative total ion current (TIC) profile of PS20 with major peaks labeled, containing sorbitan monoester, isosorbide monoester and diesters with a variety of fatty acid chains while Figure 1-V is the representative total ion current (TIC) profile of PS80 with major peaks labeled. The identified esters of PS20 and PS80 are summarized in Table 1-a and 1-b. Quantitation of polysorbates was determined by charged aerosol detector (CAD). Figure 1-II and 1-IV show the recovery of PS20 and PS80 in PS20 and PS80 standard solution and a formulated mAb by using 2D-LC/CAD respectively; the corresponding peaks were identified by mass spectrometry.

PLBD2 in Drug Substance

It has been reported that PLBD2 proenzyme (MW $64 \mathrm{kDa}$ ) was able to undergo limited autolysis leading to the formation of a $28 \mathrm{kDa}$-terminal prodomain and a $40 \mathrm{kDa}$ C-terminal mature protein (Deuschl, Kollmann, von Figura, \& Lubke, 2006), (Lakomek et al., 2009). Three different forms of PLBD2 at MW of $64 \mathrm{kDa}, 40 \mathrm{kDa}$ and $28 \mathrm{kDa}$ were all observed in the drug substance using Western blot (Figure 2, lane 2). Recombinant CHO PLBD2 also contained the three different molecular weight species (Figure 2, lane 5). Interestingly, recombinant human PLBD2 purchased from OriGene contained only proenzyme at $66 \mathrm{kDa}$ (Figure 2, lane 4).

PS20 and PS80 Degradation Pattern with human PLBD2 and CHO PLBD2

Polysorbates degradation by recombinant human PLBD2 or CHO PLBD2 was monitored as outlined in the Material and Methods section. Recombinant human PLBD2 (Figure 3-I, III) and the CHO PLBD2 (Figure 3-II, IV) with concentration at $200 \mu \mathrm{g} / \mathrm{mL}$ were incubated with $0.1 \%$ of PS20 (upper panel) and PS80 (lower panel) for 5 days. Significant PS20 degradation was observed for both human PLBD2 and CHO PLBD2 but in different patterns. By incubating PS20 with OriGene human PLBD2, a decrease in signal intensity occurred on POE-ester peaks eluting at between 27.5 and $38 \mathrm{~min}$. The peaks eluted before 34 min were POE monoester containing short fatty acid chain, i.e., POE sorbitan monolaurate, POE isosorbide monolaurate, POE sorbitan monomyristate and POE isosorbide monomyristate. The POE esters with longer chains, eluted between 34-38 min, including POE isosorbide monopalmitate, POE isosorbide monosterate and POE sorbitan diester also showed notable reduction in intensity. Higher order triesters and tetraesters, which eluted after $38 \mathrm{~min}$, did not show noticeable changes during incubation (Figure 3-I). For CHO PLBD2, similar degradation was observed in most PS20 species except the first peak representing POE sorbitan monolaurate (Figure 3-II). As for PS80 degradation, incubation with both human PLBD2 and CHO PLBD2 showed significant degradation on peaks eluting between 30 and 35 min, representing POE sorbitan monolinoleate, POE sorbitan monooleate and POE isosorbide monooleate and POE monooleate (Figure 3-III and Figure 3-IV). The CHO PLBD2 exhibited a higher degree of degradation compared to the human PLBD2. The CHO PLBD2 also showed a noticeable intensity reduction between elution time of 39 to $41 \mathrm{~min}$, corresponding to degradation POE sorbitan dioleate. POE sorbitan dioleate. The distinct degradation pattern induced by these two types of PLBD2 (human vs Chinese hamster) could be due to inherent differences between human and CHO PLBD2, or due to differences in impurities.

Monoclonal antibody expressed from PLBD2-knockout CHO cell line showed no significant reduction in co-purifying lipase activity

Polysorbate degradation was measured for monoclonal antibody drug substances produced from either control CHO cell lines or from PLBD2-knockout CHO cell lines. Drug substances were purified using Protein A and ion exchange chromatography.

The lack of PLBD2 expression in the PLBD2 knockout cell line was confirmed by Western blot analysis. The results clearly showed the clearance of PLBD2 in the knockout cell line (Figure 4-III). The representative degradation profiles of PS20 and PS80 when incubated with mAb-2 (generated by PLBD2 knockout cell line) 
are shown in Figure 4-I. The percentage of polysorbate degradation is calculated by summing changes in peak areas of monoesters (Figure 4-I). Surprisingly, the lipase activities in antibody samples expressed from the PLBD2 knockout cell line for both PS20 and PS80 were slightly higher than that from the control cell line (Figure 4-II), suggesting that PLBD2 does not play a significant role in degrading PS in drug substances. It is theoretically possible that knocking out PLBD2 in CHO cells resulted in increased expression of a different esterase, which in turn is able to degrade polysorbates. To address that possibility we performed proteomics analysis on the mAb-2 sample that was produced by PLBD2 knockout cell line. No new active lipase was found (data not shown).

\section{Depletion of PLBD2 Does Not Decreases Level of PS20 Degradation}

To further examine whether PS degradation is caused by the presence of PLBD2 in the formulated mAbs, a PLBD2 depletion experiment was performed. If PS20 degradation is caused by PLBD2, its depletion should result in diminished PS20 degradation. The relative extent of degradation should also depend on how much PLBD2 has been removed. While the knockout experiment is complicated by the possibility of additional lipase being upregulated in the PLBD2 knockout cells, the depletion experiment may provide a more direct answer as to the potential role of PLBD2 in PS degradation. Figure 5 shows the schematic workflow of the depletion experiment. An antibody against CHO PLBD2 was covalently coupled to Dynabeads for depletion of PLBD2. Specificity of the the anti-PLBD2 antibody used in this experiment was evaluated by Western blot, demonstrating that all three forms of PLBD2 present in mAb-1 including proenzyme at $64 \mathrm{kDa}$, mature protein at $40 \mathrm{k} \mathrm{Da}$ and prodomain at $28 \mathrm{kDa}$ were recognized by the anti-PLBD2 antibody (Figure 6-I lane 2). PLBD2 in mAb-1 sample can be partially (Figure 6-I lane 4) or completely depleted (Figure 6-I lane 3) by adjusting the ratio of anti-PLBD2 to mAb-1 sample during depletion. Complete depletion of PLBD2 in mAb-1 was performed by incubating $10 \mathrm{mg}$ mAb-1 with $50 \mu \mathrm{g}$ anti-PLBD2 conjugated magnetic beads while partial depletion of PLBD2 in mAb-1 was achieved by incubating $10 \mathrm{mg} \mathrm{mAb}-1$ with $10 \mu \mathrm{g}$ anti-PLBD2 conjugated magnetic beads. The percentage of PLBD2 depletion from mAb-1 was estimated by Western blot. Antibody mAb-3 which is PLBD2 free (Figure 6-I Lane 5) served as the negative control.

Prior to PLBD2-depletion, mAb-1 sample exhibited approximately $28.0 \%$ of PS20 degradation after 5 day incubation at $45^{\circ} \mathrm{C}$. After PLBD2-depletion, the PS degradation was $23.2 \%$ for partially and $27.7 \%$ for the completely depleted sample, respectively (Figure 6-II). Similar results were observed for PS80 degradation performed under the same conditions (Figure 6-III), with 18.9\% PS80 degradation observed prior to depletion, $19.32 \%$ and $20.8 \%$ PS80 degradation observed in partially and completely depleted PLBD2 samples, respectively, after 5 -day incubation at $45^{\circ} \mathrm{C}$. The lack of correlation between PLBD2 level in the samples and the extent of PS degradation in this depletion study suggested PLBD2 was unlikely the enzyme responsible for polysorbate degradation.

Amount of PLBD2 in Formulated mAb cannot be positively correlated to PS20 loss over time

To further prove that PLBD2 does not contribute to polysorbate degradation, we determined levels of PLBD2 in a number of formulated mAbs. Two PLBD2 peptides (SVLLDAASGQLR and YQLQFR) were chosen to quantitate PLBD2 in formulated mAbs using multiple reaction monitoring mass spectrometry (MRM-MS) technology. CHO PLBD2 spiked-in mAb-3 was used to create the calibration curve. Standard curves (10$500 \mathrm{ppm}$ ) with coefficients 0.9965 and 0.9943 were generated for each of the peptide (Figure 7, left panel), concentration of PLBD2 in each sample was then obtained by extrapolating it peak area onto the curve. In total, six mAbs were subjected to PLBD2 quantitation. Quantitative examination of peak areas of these 6 $\mathrm{mAbs}$ determined the concentration of PLBD2 in the formulated mAb to be between 0 to $230 \mathrm{ng} / \mathrm{mg} \mathrm{mAb}$.

PS20 degradation was then measured for the same $6 \mathrm{mAbs}$ after each sample was concentrated and buffer exchanged to $10 \mathrm{mM}$ Histidine buffer, $\mathrm{pH}$ 6. The percentage of the intact PS20 was plotted against PLBD2 concentration and correlation coefficient $\mathrm{R}^{2}$ was calculated to evaluate the linear dependence of the two variables (Figure 7 right panel). A slight downhill linear relationship with calculated Pearson correlation coefficient of 0.0042 indicates no correlation between these two variables, suggesting PLBD2 concentration in drug substances is not correlated to the PS20 loss during incubation. Among the samples tested, mAb- 
4 showed strong lipase activity with no detectable level of PLBD2, indicating another lipase/esterase was responsible for PS20 degradation in that drug substance. In a contrast, mAb-6 had high concentration of PLBD2 but showed no lipase activity, suggesting PLBD2 is unlikely the root cause of PS20 degradation.

Impurities detected and identified in commercial PLBD2 and CHO PLBD2

To explain and understand the lipase activity observed when incubating recombinant human PLBD2 or CHO PLBD2 with polysorbates, proteomics analysis was conducted to identify potential contaminant lipase(s)/esterase(s) present in the human PLBD2 and CHO PLBD2 samples. Results showed that 1600 host cell proteins were present in the human PLBD2 sample. Among these HCPs, 11 of them were proteins with potential lipase activity as listed in Table 2. One or more of those lipases may contribute to polysorbate degradation. In the CHO PLBD2 sample, 91 host cell proteins were identified, including the group XV phospholipase A2 (LPLA2), which was the lipase previously shown to degrade polysorbate by Hall, et. al(Hall et al., 2016), as suggested by high confidence identification (16 unique peptides) of this protein in our proteomics analysis (Table 3). LPLA2 was almost exclusively identified, presenting at $0.14 \%$ relative to synthetic PLBD2, and showed the exact degradation pattern of PS20 and PS80 as suggested in the literature(Hall et al., 2016).

\section{Conclusion}

PLBD2 is a host cell protein lipase present in many drug substances, A prior study suggested that PLBD2 was responsible for the degradation of polysorbates ${ }^{3}$, an enzymatic activity that at high levels, compromises drug product's quality and stability. In contrast to this notion our data indicate that PLBD2 is largely not involved in the polysorbate degradation. This conclusion is supported by three observations: PLBD2gene knockout did not reduce lipase activity; PLBD2-depleted mAb samples did not show any reduction or elimination of lipase activity; and lack of correlation between PLBD2 concentration and lipase activity. To reconcile our data and the previously observed PS degradation by purified recombinant PLBD2 we examined the purity of the recombinant PLBD2 enzymes. Several lipases were identified, raising the likelihood that the previously identified lipase activity in the recombinant human PLBD2 material was due to contaminating lipases that co-purified with human PLBD2. Our findings provide an explanation for the lack of correlation between the levels of PLBD2 in drug substances and the rates of polysorbate degradation, which has been observed by several companies in the industry. In addition, our data suggest that the clearance of PLBD2 may not be used as the sole indicator for successful removal of lipase activities in the final therapeutic protein products.

In addition to showing that PLBD2 was largely not responsible for the observed polysorbate degradation, our study offered insights that a low activity but high abundance lipase like PLBD2 could mask other active lipases present at low levels, making them less likely to be identified by common analytical methods. This study provides a novel methodology to identify other problematic host cell protein lipases, which in turn could guide the downstream purification process in developing effective strategies to remove these HCPs that may have significant impact on the therapeutic protein product quality.

\section{ACKNOWLEDGMENTS}

The authors would like to thank Andrew Tustian and Benjamin Adams of PreClinical Mfg \& Process Development for their assistance in purification of the mAbs.

\section{References}

Table 1-a. PS20 peak annotation

\begin{tabular}{ll}
\hline Peak number & Peak annotation \\
\hline 1 & POE sorbitan monolinoleate \\
2 & POE sorbitan monooleate \\
3 & POE isosorbide monooleate and POE monooleate
\end{tabular}




\begin{tabular}{ll}
\hline Peak number & Peak annotation \\
\hline 4 & POE sorbitan di-oleate \\
5 & POE isosorbide di-oleate \\
6 & POE sorbitan mixed trioleate and tetraoleate \\
\hline
\end{tabular}

Table 1-b. PS80 peak annotation

\begin{tabular}{ll}
\hline Peak number & Peak annotation \\
\hline 1 & POE sorbitan monolinoleate \\
2 & POE sorbitan monooleate \\
3 & POE isosorbide monooleate and POE monooleate \\
4 & POE sorbitan di-oleate \\
5 & POE isosorbide di-oleate \\
6 & POE sorbitan mixed trioleate and tetraoleate \\
\hline
\end{tabular}

Table 2. Lipases/ esterase identified in commercial human PLBD2

\begin{tabular}{ll}
\hline Protein Name & \# unique peptides \\
\hline $\operatorname{tr} \mid$ G3I6T1|G3I6T1_CRIGR Putative Phospholipase B-like 2 & 103 \\
$\operatorname{tr} \mid$ G3HKV9|G3HKV9_CRIGR Group XV phospholipase A2 & 16 \\
$>\operatorname{tr} \mid$ G3HQY6|G3HQY6_CRIGR Lipase & 2 \\
$\operatorname{tr} \mid$ G3HNQ5|G3HNQ5_CRIGR Phospholipase D3 & 2 \\
\hline
\end{tabular}

Table 3. Lipases/ esterase identified in CHO PLBD2

\begin{tabular}{ll}
\hline Protein Name & \# unique peptides \\
\hline tr|G3I6T1|G3I6T1_CRIGR Putative Phospholipase B-like 2 & 103 \\
tr|G3HKV9|G3HKV9_CRIGR Group XV phospholipase A2 & 16 \\
>tr|G3HQY6|G3HQY6_CRIGR Lipase & 2 \\
>tr|G3HNQ5|G3HNQ5_CRIGR Phospholipase D3 & 2 \\
\hline
\end{tabular}

\section{Figure Legends}

Figure 1. (I) Chemical structure of major expected POE esters in polysorbates, the polysorbates are mainly composed of fatty acid esters sharing common sorbitan or isosorbide head group. Lauric acid is the main fatty acid for PS20 and oleic acid is the main fatty acid for PS80. (II) CAD chromatography of PS20 standard (A) and PS20 in mAb formulation (B) separated and detected by 2D-LC-CAD, (III) Representative total ion current (TIC) profile of PS20, with major peaks labeled as POE sorbitan monolaurate (1), POE isosorbide monolaurate (2), POE sorbitan monomyristate (3), POE isosorbide monomyristate (4), POE isosorbide monopalmitate (5), POE isosorbide monosterate (6), POE sorbitan mixed diesters (7-9), POE sorbitan trilaurate and POE sorbitan tetralaurate (10). (IV). CAD chromatography of PS80 standard (A) and PS80 in $\mathrm{mAb}$ formulation (B) separated and detected by 2D-LC-CAD (V). Representative total ion current (TIC) profile of PS80, with major peaks labeled as POE sorbitan monolinoleate (1), POE sorbitan monooleate (2), POE isosorbide monooleate and POE monooleate (3), POE sorbitan di-oleate (4), POE isosorbide di-oleate and POE di-oleate (5), POE sorbitan mixed trioleate and tetraoleate (6). 
Figure 2. Western blot of PLBD2, Lane 2 is $40 \mathrm{ug} \mathrm{mAb}-1$ containing PLBD2, lane 4 is 10ng PLBD2 purchased from Origene, lane 5 is 10ng CHO PLBD2 tagged with mmHis tag (made in-house).

Figure 3. (I) Chromatogram of $0.1 \%$ PS20 in $200 \mu \mathrm{g} / \mathrm{mL}$ commercial PLBD2 spiked in $150 \mathrm{mg} / \mathrm{mL} \mathrm{mAb}-3$ incubated @ $45{ }^{\circ} \mathrm{C}$ in $10 \mathrm{mM}$ Histidine, pH6 for for 0 day (A, T0), and 5 days (B, T5). (II) Chromatogram of $0.1 \%$ PS20 in $200 \mu \mathrm{g} / \mathrm{mL}$ CHO PLBD2 spiked in $150 \mathrm{mg} / \mathrm{mL} \mathrm{mAb}-3$ incubated @ $45{ }^{\circ} \mathrm{C}$ in $10 \mathrm{mM}$ Histidine, pH6 for for 0 day (A, T0), and 5 days (B, T5). (III) Chromatogram of $0.1 \%$ PS 80 in $200 \mu \mathrm{g} / \mathrm{mL}$ commercial PLBD2 spiked in $150 \mathrm{mg} / \mathrm{mL}$ mAb-3 incubated @ $45{ }^{\circ} \mathrm{C}$ in $10 \mathrm{mM}$ Histidine, pH6 for for 0 day (C, T0), and 5 days (D, T5). (IV) Chromatogram of $0.1 \%$ PS80 in $200 \mu \mathrm{g} / \mathrm{mL}$ CHO PLBD2 spiked in $150 \mathrm{mg} / \mathrm{mL} \mathrm{mAb}-3$ incubated @ $45{ }^{\circ} \mathrm{C}$ in $10 \mathrm{mM}$ Histidine, $\mathrm{pH} 6$ for for 0 day $(\mathrm{C}, \mathrm{T} 0)$, and 5 days $(\mathrm{D}, \mathrm{T} 5)$.

Figure 4. (I) Upper panel: Chromatogram of $0.1 \%$ PS20 in $75 \mathrm{mg} / \mathrm{mL} \mathrm{mAb}-2$ (generated by PLBD2 knockout cell line) incubated @ $45{ }^{\circ} \mathrm{C}$ in $10 \mathrm{mM}$ Histidine, $\mathrm{pH} 6$ for 0 day (A, T0), and 5 days (B, T5). The percentage of PS20 degradation is calculated using equation $\% P S 20=1-\frac{\int_{27.5}^{33} I(t) \mathrm{dt} @ T 5}{\int_{27.5}^{33} I(t) \mathrm{dt} @ T 0}, \mathrm{I}(\mathrm{t})$ is the intensity of the peak at any time point. Lower panel: Chromatogram of $0.1 \%$ PS80 in $100 \mathrm{mg} / \mathrm{mL} \mathrm{mAb}-2$ incubated @ 45 ${ }^{\mathrm{o}} \mathrm{C}$ in $10 \mathrm{mM}$ Histidine, $\mathrm{pH} 6$ for 0 day $(\mathrm{C}, \mathrm{T} 0)$, and 5 days $(\mathrm{D}, \mathrm{T} 5)$. The percentage of PS20 degradation is calculated using equation $\% P S 80=1-\frac{\int_{30}^{35} I(t) \mathrm{dt} @ T 5}{\int_{30}^{35} I(t) \mathrm{dt} @ T 0}, \mathrm{I}(\mathrm{t})$ is the intensity of the peak at any time point. (II). PLBD2 knockout showed similar or higher lipase activity for PS20 and PS80 as control cell line. Upper panel: comparison of $0.1 \%$ PS20 degradation incubated with $75 \mathrm{mg} / \mathrm{mL} \mathrm{mAb}-1$ generated from control cell line at $\mathrm{pH} 6.0(\mathrm{~A})$ and $75 \mathrm{mg} / \mathrm{mL} \mathrm{mAb}-2$ generated from PLBD2 knockout cell line at $\mathrm{pH} 6.0$ (B); Bottom panel: comparison of $0.1 \%$ PS 80 degradation incubated with $100 \mathrm{mg} / \mathrm{mL} \mathrm{mAb-1} \mathrm{generated} \mathrm{from} \mathrm{control} \mathrm{cell}$ line at $\mathrm{pH} 6.0(\mathrm{C})$ and $100 \mathrm{mg} / \mathrm{mL}$ mAb-2 generated from PLBD2 knockout cell line at $\mathrm{pH} 6.0$ (D); (III). Western blot of PLBD2 in mAb-1 and mAb-2 PLBD2 knockout cell line. Lane 2 is 40ug mAb-1 and lane 3 is 40ug mAb-2 generated by PLBD2 knockout cell line.

Figure 5. Schematic diagram of the PLBD2 depletion experiment. Dynabeads magnetic beads were covalently coupled with Anti-PLBD2 monoclonal antibody and used for immunoprecipitating (IP). The original $\mathrm{mAb}(\mathrm{A})$ and flow through (B) were incubated with $0.1 \%$ PS at $45{ }^{\circ} \mathrm{C}$ for 5 days and subject to PS degradation measurement. A non-relevant antibody was served as the negative control by replacing anti-PLBD2 monoclonal antibody $(\mathrm{C})$.

Figure 6. (I) Western blot of PLBD2, Lane 1 is Mw standard, Lane 2 is $40 \mu \mathrm{g}$ mAb- 1 alone, lane 3 is $40 \mu \mathrm{g}$ mAb-1 with PLBD2 being depleted completely, lane 4 is $40 \mu \mathrm{g}$ mAb-1 with PLBD2 being partially depleted and lane 5 is $40 \mu \mathrm{g} \mathrm{mAb}-3$ containing no PLBD2. (II) The percentage of PS20 remaining in original mAb1, PLBD2-completely depleted mAb-1 and PLBD2 partially depleted mAb-1 are plotted against incubation time. The original mAb, PLBD2 completely-depleted mAb and PLBD2 partially depleted mAb are indicated by filled circle with solid line, filled diamond with dashed line and filled triangle with dotted line. (III) The percentage of PS80 remaining in original mAb-1, PLBD2-completely depleted mAb-1 and PLBD2 partially depleted mAb-1 are plotted against incubation time. The original mAb, PLBD2 completely-depleted mAb and PLBD2 partially depleted mAb are indicated by filled circle with solid line, filled diamond with dashed line and filled triangle with dotted line.

Figure 7. Left panel: calibration curve of two selected peptides YQLQFR (filled square) and SVLLDAASGQLR (filled circle) from recombinant CHO PLBD2 with mAb-3 as matrix. Right panel: correlation curve between remaining PS20 percentage and PLBD2 concentration. PLBD2 concentration were quantitated by MRM-MS using the calibration curve (SVLLDAASGQLR) in the left panel. The percentage of PS20 remaining was determined by using LC-CAD after $0.1 \%$ PS20 was incubated with various mAbs (filled circles, $75 \mathrm{mg} / \mathrm{mL}$ ) at $45{ }^{\circ} \mathrm{C}$ for 5 days.

\section{References:}

Borisov, O. V., Ji, J. A., Wang, Y. J., Vega, F., \& Ling, V. T. (2011). Toward understanding molecular heterogeneity of polysorbates by application of liquid chromatography-mass spectrometry with computeraided data analysis. Anal Chem, 83 (10), 3934-3942. doi:10.1021/ac2005789 
Cheng, Y., Hu, M., Zamiri, C., Carcelen, T., Demeule, B., Tomlinson, A., . . Yu, X. C. (2019). A Rapid High-Sensitivity Reversed-Phase Ultra High Performance Liquid Chromatography Mass Spectrometry Method for Assessing Polysorbate 20 Degradation in Protein Therapeutics. J Pharm Sci, 108 (9), 2880-2886. doi:10.1016/j.xphs.2019.04.029

Deuschl, F., Kollmann, K., von Figura, K., \& Lubke, T. (2006). Molecular characterization of the hypothetical 66.3-kDa protein in mouse: lysosomal targeting, glycosylation, processing and tissue distribution. FEBS Lett, 580 (24), 5747-5752. doi:10.1016/j.febslet.2006.09.029

Dixit, N., Salamat-Miller, N., Salinas, P. A., Taylor, K. D., \& Basu, S. K. (2016). Residual Host Cell Protein Promotes Polysorbate 20 Degradation in a Sulfatase Drug Product Leading to Free Fatty Acid Particles. $J$ Pharm Sci, 105 (5), 1657-1666. doi:10.1016/j.xphs.2016.02.029

Hall, T., Sandefur, S. L., Frye, C. C., Tuley, T. L., \& Huang, L. (2016). Polysorbates 20 and 80 Degradation by Group XV Lysosomal Phospholipase A2 Isomer X1 in Monoclonal Antibody Formulations. J Pharm Sci, 105 (5), 1633-1642. doi:10.1016/j.xphs.2016.02.022

Kiese, S., Papppenberger, A., Friess, W., \& Mahler, H. C. (2008). Shaken, not stirred: mechanical stress testing of an IgG1 antibody.J Pharm Sci, 97 (10), 4347-4366. doi:10.1002/jps.21328

Labrenz, S. R. (2014). Ester hydrolysis of polysorbate 80 in mAb drug product: evidence in support of the hypothesized risk after the observation of visible particulate in mAb formulations. J Pharm Sci, 103 (8), 2268-2277. doi:10.1002/jps.24054

Lakomek, K., Dickmanns, A., Kettwig, M., Urlaub, H., Ficner, R., \& Lubke, T. (2009). Initial insight into the function of the lysosomal $66.3 \mathrm{kDa}$ protein from mouse by means of X-ray crystallography. BMC Struct Biol, 9 , 56. doi:10.1186/1472-6807-9-56

Li, Y., Hewitt, D., Lentz, Y. K., Ji, J. A., Zhang, T. Y., \& Zhang, K. (2014). Characterization and stability study of polysorbate 20 in therapeutic monoclonal antibody formulation by multidimensional ultrahighperformance liquid chromatography-charged aerosol detection-mass spectrometry. Anal Chem, 86 (10), 51505157. doi:10.1021/ac5009628

MacLean, B., Tomazela, D. M., Shulman, N., Chambers, M., Finney, G. L., Frewen, B., . . MacCoss, M. J. (2010). Skyline: an open source document editor for creating and analyzing targeted proteomics experiments. Bioinformatics, 26 (7), 966-968. doi:10.1093/bioinformatics/btq054

Martos, A., Koch, W., Jiskoot, W., Wuchner, K., Winter, G., Friess, W., \& Hawe, A. (2017). Trends on Analytical Characterization of Polysorbates and Their Degradation Products in Biopharmaceutical Formulations. J Pharm Sci, 106 (7), 1722-1735. doi:10.1016/j.xphs.2017.03.001

McShan, A. C., Kei, P., Ji, J. A., Kim, D. C., \& Wang, Y. J. (2016). Hydrolysis of Polysorbate 20 and 80 by a Range of Carboxylester Hydrolases. PDA J Pharm Sci Technol, 70 (4), 332-345. doi:10.5731/pdajpst.2015.005942 